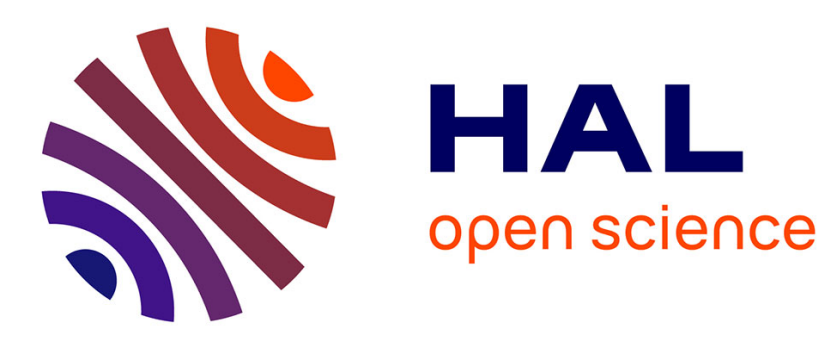

\title{
Towards multi-platform software architecture for Collaborative Teleoperation
}

Christophe Domingues, Samir Otmane, Frédéric Davesne, Malik Mallem

\section{To cite this version:}

Christophe Domingues, Samir Otmane, Frédéric Davesne, Malik Mallem. Towards multi-platform software architecture for Collaborative Teleoperation. 2nd Mediterranean Conference on Intelligent Systems and Automation (CISA'09), Mar 2009, Zarzis, Tunisia. pp.347-349, 10.1063/1.3106500 . hal-00376597

\section{HAL Id: hal-00376597 https://hal.science/hal-00376597}

Submitted on 18 Apr 2009

HAL is a multi-disciplinary open access archive for the deposit and dissemination of scientific research documents, whether they are published or not. The documents may come from teaching and research institutions in France or abroad, or from public or private research centers.
L'archive ouverte pluridisciplinaire HAL, est destinée au dépôt et à la diffusion de documents scientifiques de niveau recherche, publiés ou non, émanant des établissements d'enseignement et de recherche français ou étrangers, des laboratoires publics ou privés. 


\title{
Towards multi-platform software architecture for Collaborative Teleoperation
}

\author{
Christophe Domingues*, Samir Otmane*, Frederic Davesne* and Malik Mallem* \\ *Informatics, Integrative Biology and Complex Systems, \\ 40 Rue de Pelvoux, 91020 Evry Cedex, France (e-mail: name.familyname@ibisc.fr).
}

\begin{abstract}
Augmented Reality (AR) can provide to a Human Operator (HO) a real help in achieving complex tasks, such as remote control of robots and cooperative teleassistance. Using appropriate augmentations, the HO can interact faster, safer and easier with the remote real world. In this paper, we present an extension of an existing distributed software and network architecture for collaborative teleoperation based on networked human-scaled mixed reality and mobile platform. The first teleoperation system was composed by a VR application and a Web application. However the 2 systems cannot be used together and it is impossible to control a distant robot simultaneously. Our goal is to update the teleoperation system to permit a heterogeneous collaborative teleoperation between the 2 platforms. An important feature of this interface is based on the use of different Virtual Reality platforms and different Mobile platforms to control one or many robots.
\end{abstract}

Keywords: Augmented Reality, Virtual Reality, Telerobotic, Teleoperation, Collaboration, Architecture, Robot.

\section{INTRODUCTION}

$\mathrm{E}$ arly Computer Assisted Teleoperation architectures provided to the human operator many kinds of assistances. However diverse assistance strategies and information's concerning tasks are proposed and the robot commands where a function of operator actions and the adopted strategy (e.g Freezing some robot Degrees of freedom). The problem with this approach is that the current solutions are used in closed platforms and do not allow changes of functionalities. Virtual Reality (VR) and Augmented Reality can benefit from teleoperation systems to improve human robot interaction. VR/AR has been used to solve time delay problems using predictive display concept where a virtual robot is surrounding the real robot on the video feedback [1][2].

In our laboratory, we have worked several years on human machine interaction model for teleoperation systems that led to the ARITI system. ARITI permits to control a 6 DoF robot with the use of Virtual Reality and Augmented Reality. Two teleoperation platforms exist. The first using a Virtual Reality/Augmented Reality semi immersive platform [1][2] and the second is an online version accessible through a Web browser. Recently, collaboration functionalities have been integrated on the VR platform and Web platform [3][4]. However, technical problems and utilization limitations appears that are described on this paper. The major problem is that the 2 platforms cannot be used at the same time. Consequently, collaboration is not possible between the 2 platforms. Collaborative teleoperation has been motivated by several situations: physical limitation of manipulator's workspace or grasping large objects. With this work, our final goal is to permit collaborative teleoperation (users-robots) with multiple interfaces and multiples devices (see fig 1). So we want to make possible collaboration between VR/AR users, Mobile users and distant robots.

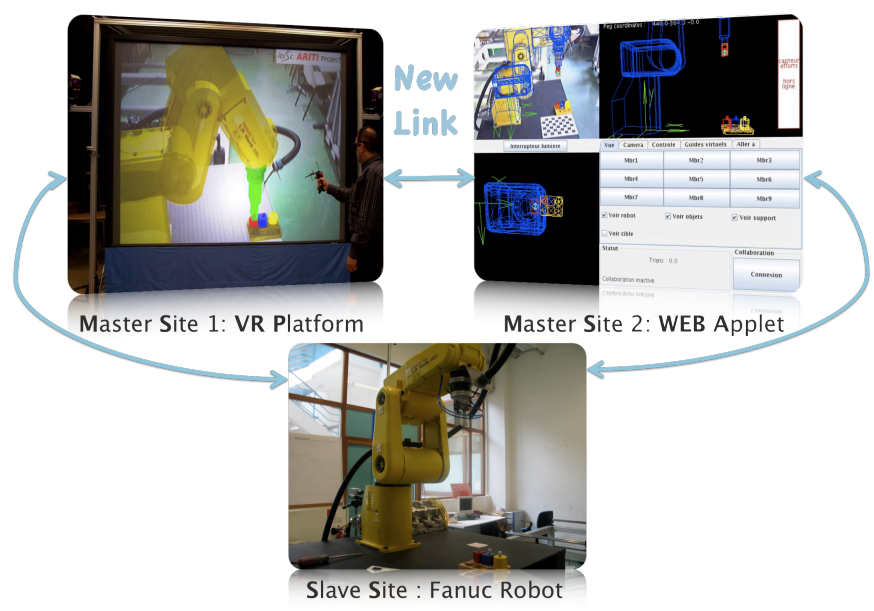

Fig. 1: Illustration of the collaborative teleoperation between VR and WEB interfaces

\section{THE COLLABORATIVE TELEOPERATION SYSTEM}

\subsection{A new Network Architecture}

To remedy the limitation of the non-use of the 2 platforms together, we have added the multi user server. This server permits to link VR and Mobile applications. We choose a central server instead of a peer-to-peer architecture to prevent from synchronisation problems. With a central server, resources are available only at a moment in the same machine. Figure 2 shows the network architecture with the integration of the multi user server. This server is connected to a MySQL database storing position and orientation of all shareable objects but also the position and orientation of the users' virtual robots. The multi user server is connected to the robot server to transmit the robot commands from the users. 


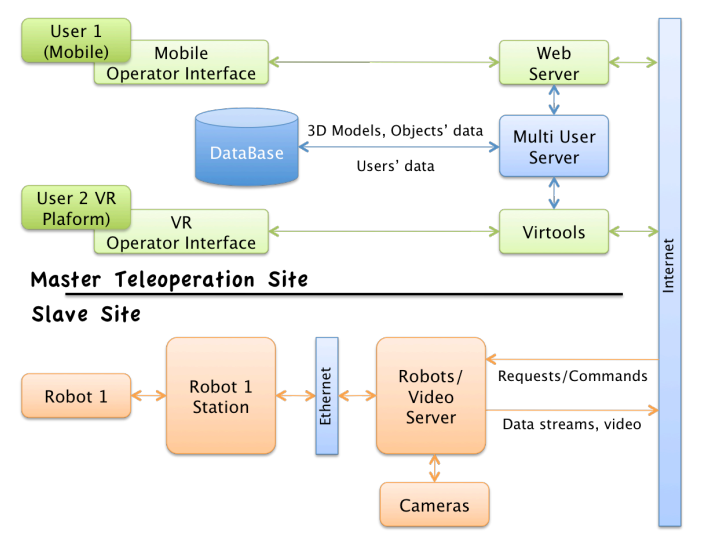

Fig. 2: The network architecture for the new teleoperation system. The user can indifferent used a Web or VR application.

For the Video component, we have used Windows Media Services based on Windows Server 2003 Enterprise Edition on the relay server. The relay server receives streams from different servers coder and diffuses (Multicast or Unicast) to the connected clients. The encoder servers used Windows Media Coder 9. This solution has been chosen instead of ffmpeg streaming solution because streaming with ffmpeg does not work correctly with Virtools. Figure 3 shows the proposed streaming architecture. The media coder can encode video and audio from different sources only the sources are DxShow compatible (e.g. Webcams or Firewire cameras). For the moment, we have not performed tests to choose the best encoding format.

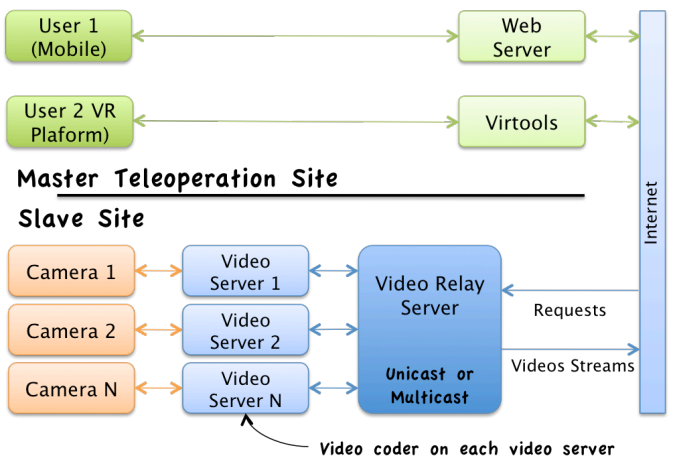

Fig. 3: Zoom on the streaming network architecture

\subsection{A new Software architecture}

With this work, we wanted to integrate on the software architecture a system that will permit the personalization of the application. To achieve this objective, we have considered all the functionalities as modules where each module is designed with the same conventions and is available in 2 versions (Mobile and classic). A module has 2 ways of working (Safe or classic mode) and communicates with the core. There are 2 cores version, the mobile and the VR Core. The VR core is implemented in Virtools scripting language while the second is implemented in JAVA. Despite of the existence of 2 core versions, each core has the same structure, functions, inputs and outputs. The core sends to a module synchronization signals as "LOAD" or "UNLOAD".
These signals are used to load the module or unload the module when the application is running. At the beginning, modules are loaded by the module loader (component of the core). When the load is complete, the module gives a state report to the core that permits to determine the working mode (Safe or classic). A safe mode permits to activate some possibilities of the module by sending an adequate signal ("SAFE"). The core also sends with the signal an argument. This argument is a degree of degradation. For example, the user has selected 5 cameras to get 5 point of view of the distant robot but the latency is too high. So this core will automatically use the maximum number of cameras possible to have a good latency in order to preserve a good experience for the user, the module receives "SAFE 4" with the argument " 4 " for 4 cameras. Figure 4 shows the software architecture with the used of 3 modules.

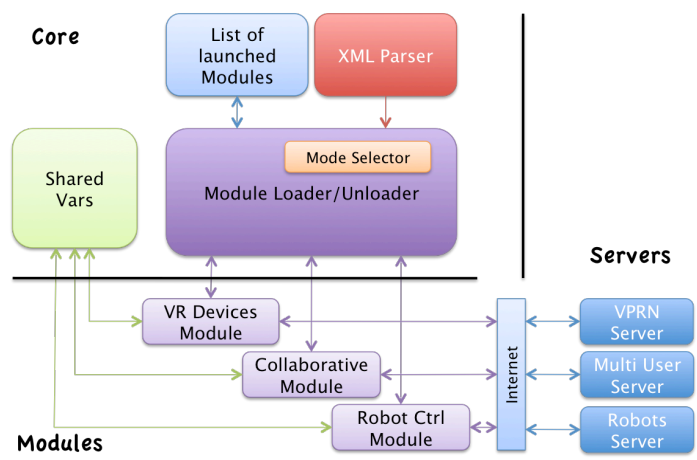

Fig. 4: Software architecture example with 3 modules for VR application. The core is the same for both platforms but modules are adapted to the support.

The main advantage of this architecture is that the user can dynamically add modules in the application without stopping it. For example, during a teleoperation task the user has initially chosen to free control the robot, however he needs to program a trajectory and stored it in the robot. In this case, the system is available to add the trajectory module when the application is running. The prototyping system uses the web server of the mobile applications. The user will use the Prototyping System to select all the elements he wants to use in the teleoperation application. In fact, a module is a package, which contains the module and an XML description of the module, which is containing methods and arguments. A final XML file is created which is containing all modules description and the user option.

\section{CONCLUSION AND PERSPECTIVES}

We presented a new collaborative Man Machine Interface (MMI) allowing high-level teleworking activities and collaborative robot teleoperation using Augmented and Virtual Reality technologies. We started this work by identifying problems and limitations on actual systems. In fact, we wanted to integrate collaborative robot-teaching aspects with the support of multi interfaces and multi devices. Consequently, we have built our architecture with this constraint. One of the major problems encountered was the video streaming to permit a synchronous diffusion of the distant robot video stream. The second condition is to permit 
to quickly and easily add/delete/update modules on the system. To answer these conditions we have proposed a Prototyping System, which permit to personalize an application by clicking on desired modules. PS permits to choose between a VR application compatible with the platform and a Web application usable on a Web Browser or any mobile device. Future work will focus on many aspects as:

- Completing the transformation of the old ARITI system by developing missing modules;

- Performing evaluations on the interaction aspects (Mobile versus VR; Virtual versus Real, VR versus VR) with the use of an evaluation system to improve the system;

- A new model of Virtual Fixtures as it exists on ARITI but for a collaborative case where users want to grasp the same object at the moment.

\section{REFERENCES}

[1] S. Otmane, M. Mallem, A. Kheddar, and F. Chavand. Ariti: an augmented reality interface for teleopeation on the Internet. In Advanced Simulation Technologies Conference, pages 254-261. Wyndham City Center Hotel, Washington, D.C. (USA), April 12-20 2000.

[2] C. Domingues, S. Otmane, F. Davesne M. Mallem and L. Benckick. A Distributed Architecture for
Collaborative Teleoperation using Virtual Reality and Web Platforms. IEEE CCNC 2009, Las Vegas, (USA)

[3] S. Otmane, M. Mallem, A. Kheddar and F. Chavand. Active virtual guide as an apparatus for augmented reality based telemanipulation system on the Internet. In IEEE Computer Society, 33rd Annual Simulation Symposium ANSS 2000, pages 185-191, Wyndham City Center Hotel, Washington, D.C (USA), 2000.

[4] V. Leligeour, S Otmane, P. Richard, and M. Mallem Distributed Software Architecture for Collaborative Teleoperation based on Networked Mixed Reality Platforms. In 2nd IEEE Int. Conf. on Information \& Communication Technologies: From Theory to Applications, pages 3498-3503, Damascus (Syria), 24-28 April 2006. 